\title{
Quantum Dot-Based Molecularly Imprinted Polymers on Three- Dimensional Origami Paper Microfluidic Chip for Fluorescence Detection of Phycocyanin
}

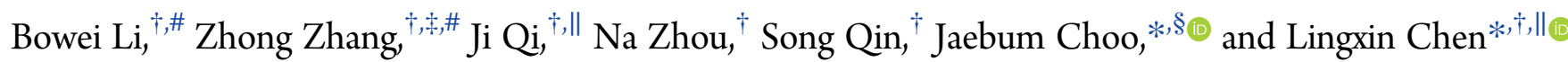

${ }^{\dagger}$ Key Laboratory of Coastal Environmental Processes and Ecological Remediation, Yantai Institute of Coastal Zone Research, Chinese Academy of Sciences, Yantai 264003, China

ॠCollege of Food Engineering and Nutritional Science, Shaanxi Normal University, Xi'an 710119, China

${ }^{\S}$ Department of Bionano Engineering, Hanyang University, Ansan 426-791, South Korea

"School of Environment and Materials Engineering, Yantai University, Yantai 264005, China

Supporting Information
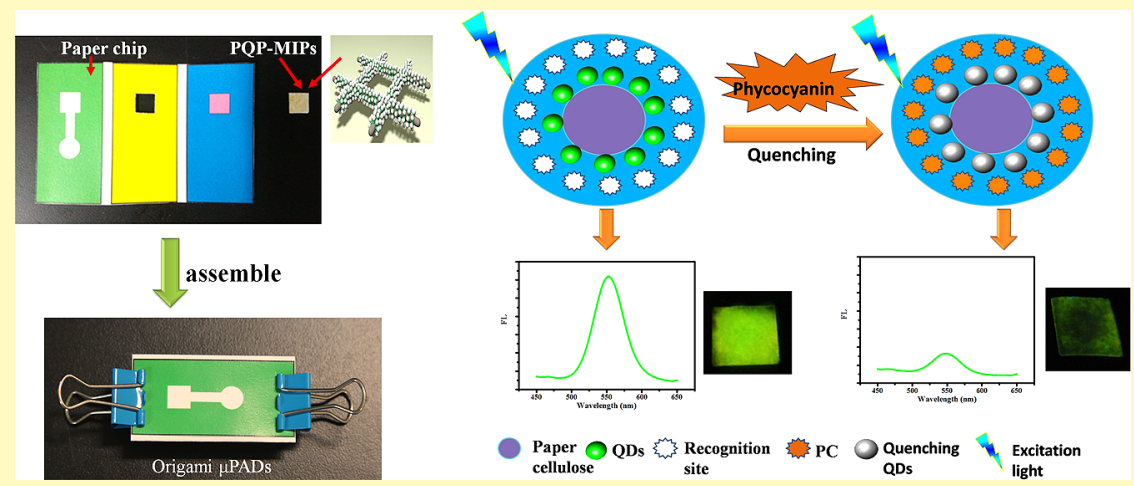

ABSTRACT: In this work, we developed a novel strategy using fluorescent quantum dots (QDs) combined with molecularly imprinted polymers (MIPs) on three-dimensional (3D) origami paper-based microfluidic devices for specific recognition and sensitive detection of phycocyanin. This method can realize the liquid phase of QDs@MIPs being transferred to the solid-phase paper base and achieve easy portability for the analysis. Under optimal conditions, we successfully demonstrated the proposed paper@QDs@MIPs 3D microfluidic chip for the sensitive and selective detection of phycocyanin protein target in a simple and robust manner. Our results revealed that the method exhibited a dynamic response to phycocyanin in the range of $10-50 \mathrm{mg} / \mathrm{L}$ with a limit of detection of $2 \mathrm{mg} / \mathrm{L}$. Importantly, this device could provide quantitative information very conveniently and show great potential to be further extended to the detection of other proteins or biomarkers for environmental and food safety research.

KEYWORDS: paper-based microfluidic devices, molecularly imprinted polymers (MIPs), quantum dot, phycocyanin, fluorescence

$\mathrm{P}$ hycocyanin, a blue-colored photosynthetic accessory pigment protein, is widely distributed in seawater, lake water, and algae microbes in drinking water, and has good survival ability. In addition, phycocyanin is a new desirable fluorescent marker with high stability and sensitivity and could be applied in biomedical and environmental fields. In the case of eutrophication of the water environment it often leads to algae bloom, causing serious deterioration of water quality. ${ }^{1-3}$ The content of toxic substances produced by blue-green algae, including $\beta$-methylamino-alanine, ${ }^{4}$ algal toxins, ${ }^{5}$ nodularin, ${ }^{6}$ and saxitoxin, $2,7,8$ in the water is also increased and represents a serious threat to the safety of marine life and humans. Therefore, to protect the ecological balance of the environment, it is necessary to monitor and control the quantity of cyanobacteria. As its concentration can effectively represent cyanobacterial biomass, it can be used as an index of cyanobacteria outbreak and plays an important role in monitoring the marine ecological environment. ${ }^{9}$ Current methods of detecting phycocyanin concentration mainly involve remote sensing methods, ${ }^{10,11} \mathrm{UV}$, and fluorescence detection. ${ }^{12,13}$ In addition, direct analysis of the water content of phycocyanin often requires separation and purification steps. Moreover, high cost and time-consuming methods add to the challenge, and development of a low-cost, fast, and efficient method for the detection of phycocyanin is urgently needed.

Since the concept of microfluidic paper-based analytical devices ( $\mu$ PADs) was first proposed by the Whitesides group, ${ }^{14}$ versatile $\mu \mathrm{PADs}$ have recently attracted increasing atten-

Received: October 24, 2016

Accepted: January 25, 2017

Published: January 25, 2017 
tion. ${ }^{15,16}$ This approach combines several great advantages such as miniaturization, automation, and integration. Moreover, compared with traditional materials such as PDMS and glass, it could present superior features such as low cost, ease of use, and facile portability, and operation. Hence, a large number of techniques including colorimetric, ${ }^{17}$ electrochemical, ${ }^{18-20}$ chemiluminescence, ${ }^{21}$ surface enhanced Raman spectroscopy (SERS) sensors, ${ }^{22-24}$ and biochemical analyses ${ }^{25-27}$ have been reported, mainly focusing on point-of-care (POC), ${ }^{28-31}$ biochemical tests, ${ }^{32,33}$ and environmental monitoring. ${ }^{34,35}$

Molecularly imprinted polymer (MIP) technologies concern creation of tailor-made binding sites in a polymer matrix with memory of the shape, size, and functional groups of the template molecules, and provide unique advantages such as excellent recognition specificity, high selectivity, and good reusability. ${ }^{36,37}$

Therefore, the combination of the MIP technique with paper-based microfluidics provides potential reproducible results for the quantitative measurement of analytes. Recently some emerging techniques have used molecularly imprinted polymers and quantum dots (QDs) techniques on a paperbased platform. Noor and Krull developed a fluorescence resonance energy transfer system by immobilizing multicolor QDs as donors. ${ }^{38}$ Our group proposed $\mathrm{SiO}_{2} @ \mathrm{QDs} @ m s-M I P s$ and magnetic micromotor-driven sensor strategies for detection of phycocyanin. ${ }^{12,39}$ Yu's group presented a paper-based MIPgrafted multidisk plate for chemiluminescence detection through an indirect competitive assay ${ }^{40}$ and developed a photoelectrochemical sensor by immobilizing QDs@MIPs on screen-printed working electrodes. ${ }^{41}$ However, there is still a lack of strategies for grafting MIPs and quantum dots directly on the paper to realize simple and facile detection.

To address these issues, we presented a new kind of quantum dot wrapped with molecularly imprinted polymers on $3 \mathrm{D}$ origami paper-based microfluidic chip devices that allow direct monitoring of phycocyanin. To the best of our knowledge, the quantum dot was first grafted on the paper through synthesizing the paper@QDs@PC-MIPs component and realizing the liquid phase of QDs@MIPs being transferred to the solid phase paper base. The paper@QDs@PC-MIPs component could be easily assembled into the 3D origami paper-based devices, and it provides a simple, portable, and user-friendly way for phycocyanin detection with desirable results.

\section{EXPERIMENTAL SECTION}

Materials. Phycocyanin (PC) powder was supplied by Shandong Oriental Ocean Company (Yantai, China). Sodium borohydride, cadmium chloride, N-hydroxysuccinimide (NHS), and 1-ethyl-(3(dimethylamino)propyl) carbodiimide hydrochloride (EDC) were purchased from Aladdin Industrial Corporation (Shanghai, China). Albumin from chicken egg, bovine serum albumin (BSA), and 3aminopropyl triethoxysilane (APTES) were obtained from SigmaAldrich Corporation (Shanghai, China). Hemoglobin from bovine blood (BHB) was purchased from Solarbio. Thioglycollic acid (TGA), ethanol, phosphate buffered saline (PBS), and other chemical reagents were of at least analytical grade and were purchased from Sinopharm Chemical Reagent (Shanghai, China). Whatman No. 1 filter paper was purchased from GE (Shanghai, China). All aqueous solutions used throughout the work were prepared with ultrapure water (18.2 $\mathrm{M} \Omega$ ), which was produced by a Pall Cascada laboratory water system (Millipore, Bedford, MA, USA).

Synthesis of Water-Soluble CdTe QDs. Water-soluble TGAmodified CdTe QDs were synthesized according to the reported method. ${ }^{42}$ To form the cadmium precursor, $68.4 \mathrm{mg}$ of $2 \mathrm{CdCl}_{2} \cdot 5 \mathrm{H}_{2} \mathrm{O}$,
$63 \mu \mathrm{L}$ of TGA, and $75 \mathrm{~mL}$ of highly purified water were mixed in a three-necked flask. The mixture was adjusted to $\mathrm{pH} 9-9.2$ with $1.0 \mathrm{M}$ $\mathrm{NaOH}$ and stirred under $\mathrm{N}_{2}$ for $20 \mathrm{~min}$. Meanwhile, $38.3 \mathrm{mg}$ tellurium powder, $40.0 \mathrm{mg} \mathrm{NaBH}, 1.5 \mathrm{~mL}$ ethanol, and $0.5 \mathrm{~mL}$ highly purified water were mixed in a sealed $2 \mathrm{~mL}$ tube. The solution was heated at 40 ${ }^{\circ} \mathrm{C}$ for $4 \mathrm{~h}$ to format $\mathrm{NaHTe}$ solution and then $1 \mathrm{~mL}$ of freshly prepared NaHTe aqueous solution was injected into the reaction system under stirring. The solution was heated until boiling and refluxed for $2 \mathrm{~h}$. The resultant TGA-capped CdTe QDs exhibited strong yellow-green fluorescence at $545-560 \mathrm{~nm}$. CdTe Quantum dot solution was stored in the dark and its fluorescence intensity did not change significantly over 60 days.

Synthesis of Fluorescent Sensors Filter Paper Substrate (Paper@QDs). The filter paper substrate was cut to a size of $8 \mathrm{~mm} \times$ $8 \mathrm{~mm}$, dipped in $0.2 \mathrm{~mol} / \mathrm{L} \mathrm{HCl}$ solution for $30 \mathrm{~min}$, and washed three times with ultrapure water to remove excess $\mathrm{HCl}$. The paper was immersed in $20 \mathrm{~mL}$ of ethanol/water $(50 \%)$ and $200 \mu \mathrm{L}$ of APTES was added for 2-4 h for addition of receiving amino groups. After removal of excess alcohol by DDI water, the paper substrate was retained and set aside. A total of $10 \mathrm{~mL}$ CdTe quantum dots was slowly added dropwise to the filter paper substrate, and then $6 \mathrm{~mL}$ of $20 \mathrm{mg} / \mathrm{mL}$ EDC (1-ethyl-3-(3-(dimethylamino)propyl) carbodiimide) and $6 \mathrm{~mL}$ of $10 \mathrm{mg} / \mathrm{mL}$ NHS (N-hydroxysuccinimide) solution were added and reacted by oscillation at room temperature in the dark for 8 $\mathrm{h}$ to allow grafting of the quantum dots at the surface of the paper substrate (shown in Figure. 3). Finally, the product was rinsed 3-5 times in $0.01 \mathrm{~mol} / \mathrm{L}$ phosphate buffer solution $(\mathrm{pH}=7.5)$ and ultrapure water.

Synthesis of Paper@QDs@PC-MIPs (PQP-MIPs) Structure. The PQP-MIPs were prepared by a surface imprinting process similar to reported procedures with some modifications. ${ }^{43}$ To form the complex, $5 \mathrm{mg}$ phycocyanin and $40 \mu \mathrm{L}$ APTES were added to $20 \mathrm{~mL}$ highly purified water, followed by addition of $50 \mu \mathrm{L}$ TEOS as crosslinker and $50 \mu \mathrm{L}$ ammonia oscillation at $80-100 \mathrm{rpm} / \mathrm{min}$ in a rotary shaker for $30 \mathrm{~min}$ to allow polymerization. The prepared paper@QDs was incubated in the polymerization solution in the dark for $12 \mathrm{~h}$ to graft the phycocyanin-MIPs. The resultant PQP-MIPs was rinsed three times to remove residues. A $1 \%$ Triton X-100 solution was used as the eluent to remove excess phycocyanin from the PC-MIPs. For comparison, nonimprinted polymers paper@QDs@NIPs (hereafter called NIPs for simplicity) were prepared as a control using the same procedure and conditions, but in the absence of phycocyanin. In this work, the paper@QDs have a covalent bond that makes the quantum dots graft on the paper firmly, and holds the signal stable. To prove this, the fluorescence intensity investigations of QDs on paper (covalently immobilized or physically adsorbed) before and after rinsing were carried out (as shown in Figure S1). It can be observed that the fluorescence intensity was very weak for the QDs only depending on the physical adsorption (curve c) compared with the covalently immobilized QDs (curve a) on the paper. In addition, after the rinsing, the fluorescence intensity of covalently immobilized QDs did not change significantly (curve b) and it indicated that the covalent bond made the quantum dots graft on the paper firmly, and maintained the signal high and stable.

Instruments. The chip was designed with drawing software (Adobe Illustrator) and then directly printed onto the filter paper (Whatman chromatography No. 1 paper, GE) by a wax printer (XEROX Phaser 8560DN) with maximum resolution of 2400 dpi. After printing, the paper was kept in an oven at $150{ }^{\circ} \mathrm{C}$ for $20 \mathrm{~s}$ to let the wax penetrate through the paper completely, forming a hydrophobic barrier as described in previous reports. ${ }^{18,22}$

Modifications of the paper were imaged by scanning electron microscopy (SEM; JSM $5600 \mathrm{LV}$, operating at $20 \mathrm{kV}$ ). Fluorescence spectra were obtained using a spectrofluorometer (Fluoromax-4, HORIBA): excitation light was set at $396 \mathrm{~nm}$ and the emission spectra were recorded from 500-650 nm for QDs. A fluorescence microscope (IX51, Olympus) equipped with a CCD camera was used to observe the fluorescence microscopy image on a glass slide. Infrared spectra were obtained from Fourier transform infrared spectrometer (Nicolet iS10, Thermo Scientific) to examine the preparation process. UV/vis 
absorption spectra were recorded on a spectrophotometer (NanoDrop2000/2000C, Thermo Scientific) to test the binding properties of polymers.

\section{RESULTS AND DISCUSSIONS}

Fabrication and Characterization of Paper@QDs@PCMIPs Base Component. Figure 1 schematically illustrates the

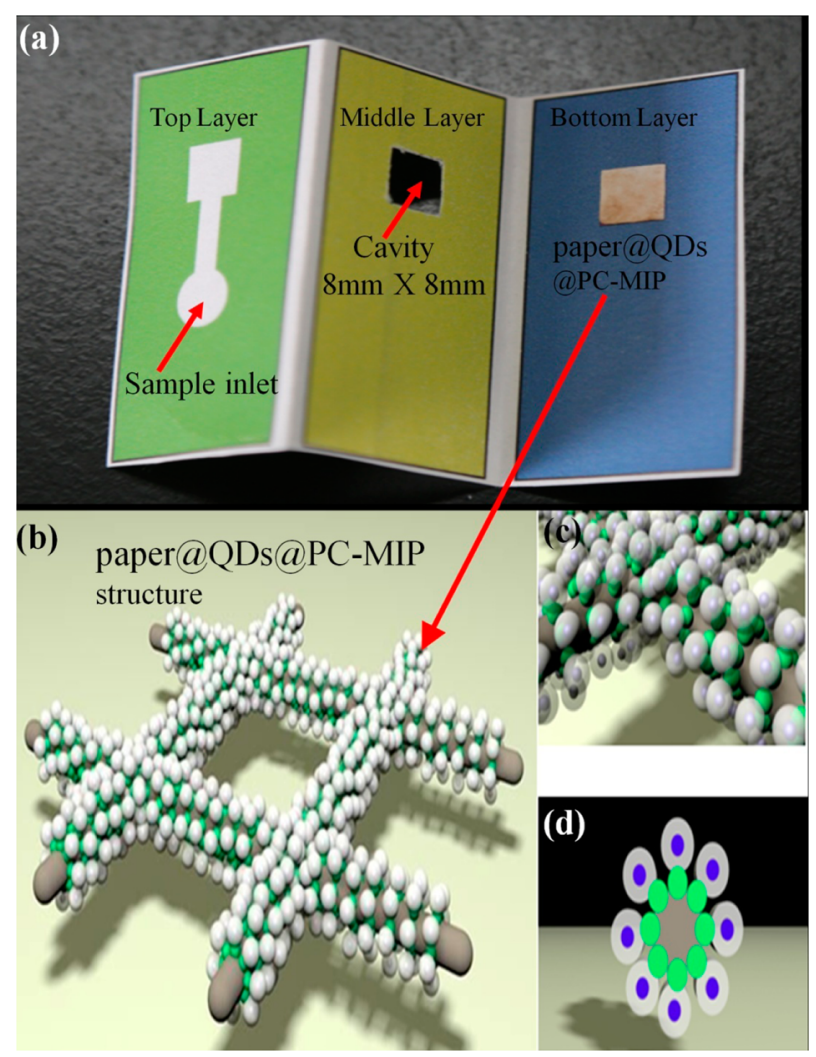

Figure 1. (a) Photograph of the paper@QDs@PC-MIPs $\mu$ PADs. (b) Schematic illustration of the structure of the paper@QDs@PC-MIPs component. (c,d) Side and bottom view of enlarged diagram of the structure of the paper@QDs@PC-MIPs on cellulose paper. Green shell: fluorescent quantum dots; white shell: PC imprinting; gray column: cellulose paper.

structure and the sensing principle of paper@QDs@PC-MIPs base component. The filter paper substrates were used as base materials for the main component of the $\mu \mathrm{PADs}$ because paper is composed of a $3 \mathrm{D}$ cellulose fiber that provides a good supporting base and the hydroxyl group of cellulose can be easily modified. The paper component was cut into a square piece of $8 \mathrm{~mm} \times 8 \mathrm{~mm}$. This bare paper was first bathed in a $0.2 \mathrm{M}$ hydrochloride solution for $30 \mathrm{~min}$ to activate the abundant $\mathrm{OH}$ groups on each hydroglucose unit of the filter paper. Next, $200 \mu \mathrm{L}$ APTES was added to a $20 \mathrm{~mL}$ mixture of ethanol and water $(50 \%, \mathrm{v} / \mathrm{v})$ and the paper was immersed in this solution for 2-4 h to graft amino groups onto the paper. Excess amino was then removed by rinsing three times with ultrapure water. The fluorescent filter paper substrate was fabricated using an amidation reaction between the amino groups on the paper and the carboxyl groups of TGA-capped CdTe QDs. Solutions of $20 \mathrm{mg} / \mathrm{mL}$ EDC and $10 \mathrm{mg} / \mathrm{mL}$ NHS were prepared with MES buffer $(\mathrm{pH}=5.2)$, and $10 \mathrm{~mL}$ TGAcapped CdTe QDs, $6 \mathrm{~mL}$ EDC, and $6 \mathrm{~mL}$ NHS were thoroughly mixed by ultrasonication for approximately $10 \mathrm{~min}$.
The paper with abundant amino groups was immersed in this solution and incubated in the dark at room temperature for 12 $\mathrm{h}$ to yield the fluorescent CdTe QDs filter paper substrate (QDs @paper). Next, the functional monomer (APTES), template molecule (phycocyanin), cross-linker (TEOS), and catalyst $\left(\mathrm{NH}_{3} \cdot \mathrm{H}_{2} \mathrm{O}\right)$ were added to form the imprinted silica shell on the surface of paper@QDs. In the presence of phycocyanin, a Meisenheimer complex would be produced between phycocyanin and amino groups on the CdTe QDs of the paper fiber; hence, the photoluminescent energy of QDs would be transferred to the complex and result in QD fluorescence quenching (shown in Figure 2). When the template molecule is removed, the fluorescence intensity is restored.

To evaluate the modified morphologic structures of the paper, images of bare filter paper substrate, paper@QDs, and paper@QDs@MIPs were obtained by SEM. As shown in Figure $3 \mathrm{a}$, the filter paper showed a nonuniform internal cellulose structure. Figure $3 \mathrm{~b}$ shows that the cellulose paper surface wrapped with the quantum dots had a highly uniform spherical morphology, illustrating that CdTe quantum dots had been grafted on the paper fiber surface and that these CdTe quantum dots were evenly distributed on the cellulose paper. As shown in Figure 3c, the surface had an obvious molecular imprinting layer, modified by the sol gel method. After modification (as shown in Figure 3e), the imprinting paper chips still had good fluorescence properties. Figure S2 showed FT-IR spectra of bare paper, paper@QDs, and paper@QDs@ PC-MIPs samples, respectively. The results revealed that QDs and MIPs were grafted successfully.

Fabrication of 3D Microfluidic Paper-Based Chip Device. The three-dimensional PQP-MIPs $\mu$ PADs design is shown in Figure S3. Briefly, the dimensions of the chip were 5.5 $\mathrm{cm} \times 2.7 \mathrm{~cm}$, the sample reservoir was $6 \mathrm{~mm}$ in diameter, and the distance between the sample reservoir and the detection area $\left(8 \times 8 \mathrm{~mm}^{2}\right)$ was $1.2 \mathrm{~cm}$. The 3D $\mu$ PADs were composed of three layers (Figure S4, in Supporting Information). The top layer was set with a central sample reservoir connected to fluid channels that led to the paper@QDs@PC-MIPs component at the bottom layer. The wax hydrophobic barrier helped the fluid wick from the sample reservoir into the detection area by capillary forces. The middle layer was printed with wax in advance, and then an $8 \times 8 \mathrm{~mm}^{2}$ cavity was gauged by the knife, cutting to fit for assembly of the paper@QDs@PC-MIPs component, as discussed above. The third layer was fully patterned with wax and then attached to the paper@QDs@PCMIPs component (shown in Figure S4a,b) to maintain good contact with the square area of the top layer. Finally, the 3D $\mu$ PADs were assembled using the clamps (Figure S4c). The 3D origami $\mu \mathrm{PADs}$ are very simple and easy to fabricate. Moreover, some water samples are not clean and require the filtration of the pretreatment process, as shown in Figure S5, when the phycocyanin sample flowed through the channel and reached the paper@QDs@PC-MIPs, the Meisenheimer complex was generated between the phycocyanin and the amino groups on the CdTe QDs, resulting in energy transfer to the complex with quenching of QDs fluorescence. Light at wavelength $396 \mathrm{~nm}$ excited the fluorescence of paper@QDs@PC-MIPs that was set through the solid sample holder, and the fluorescence spectra of PQP-MIPs $\mu$ PADs were recorded using a spectrofluometer (Fluoromax-4, HORIBA). It was noticed that the fluorescence intensity of the paper chip recovered remarkably after eluting three times, proving that the elution of the template molecule 


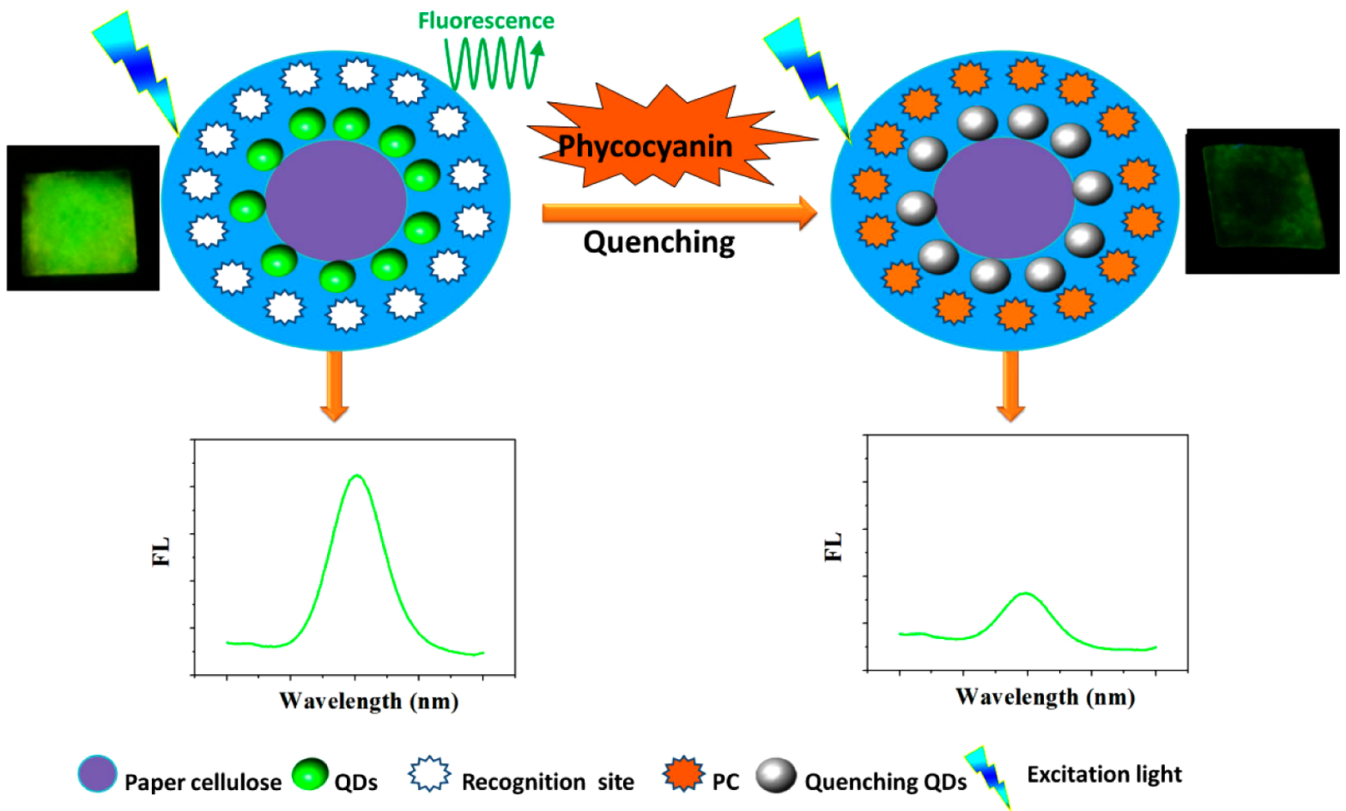

Figure 2. Schematic illustrations of the structure and the sensing principle of paper@QDs@PC-MIPs.

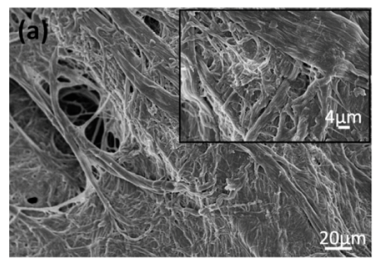

(b)
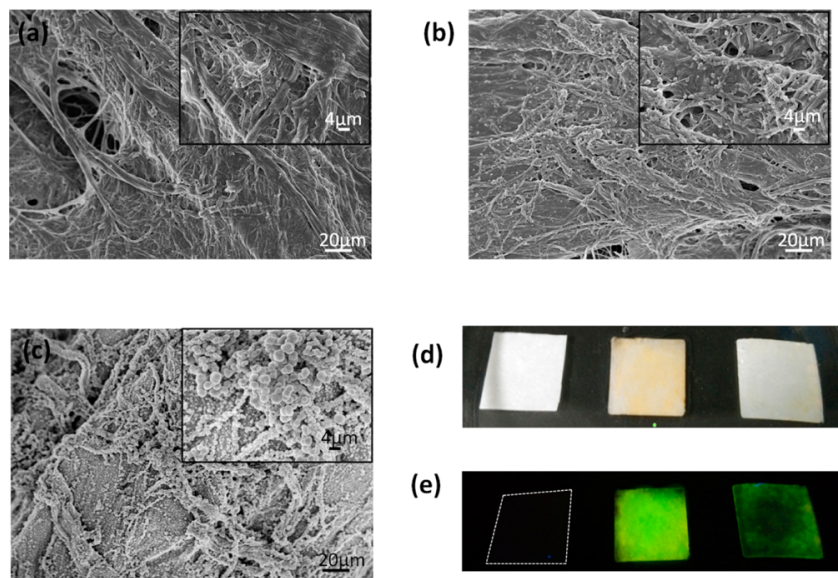

(d)

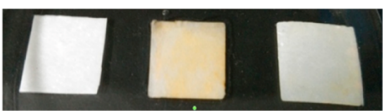

(e)

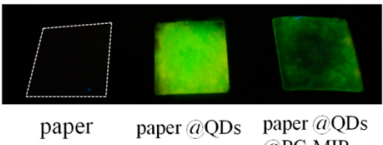

aPC-MIP

Figure 3. (a-c) SEM images of filter paper, paper@QDs, and paper@ QDs@PC-MIPs; scale bars are $20 \mu \mathrm{m}$ and $2 \mu \mathrm{m}$, respectively. (d,e) Photographs of filter paper, paper@QDs, and paper@QDs@PC-MIPs under room light $(\mathrm{d})$ and ultraviolet lamp (e). was complete without interfering with the fluorescence (as shown in Figure S6).

Detection of Phycocyanin. The amino groups of functional monomer (APTES) molecules and the carboxyl groups of phycocyanin have a strong hydrogen bonding interaction (Figure S7). QDs for protein detection are mainly based on the electronic transfer caused by fluorescence quenching and surface adsorption. The amination reaction was carried out by APTES through covalent $\mathrm{Si}-\mathrm{O}$ bonds with the hydroxyl groups of cellulose on the surface of the paper fiber. As illustrated in Figure 2, if there is no template molecule on the surface of QDs, the electrons of QDs can accept the UV energy and will be excited. Subsequently, when the excited electrons return to the ground state the quantum dot will emit green fluorescence. It is important to note that if the energy level of phycocyanin is higher than that of CdTe quantum dots, when the excited electrons of QDs return to the ground state, they will not generate fluorescence. Therefore, adsorption of more phycocyanin molecules onto the surface imprinting sites will result in greater fluorescence quenching. We further investigated the PC dynamic equilibrium curve that illustrated
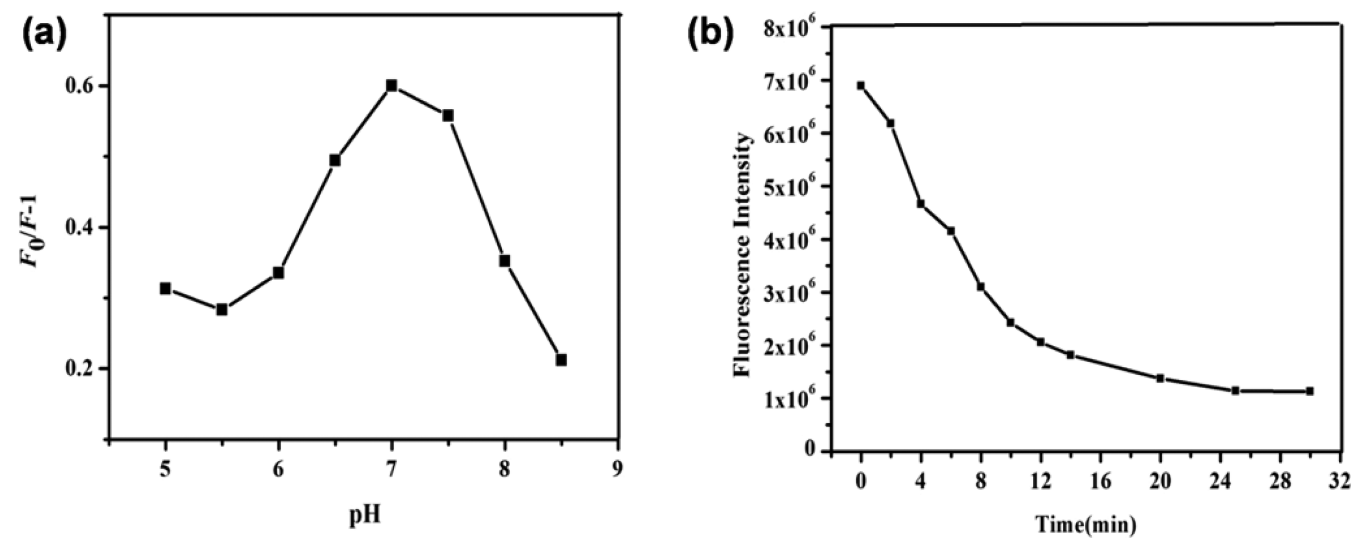

Figure 4. (a) Effect of pH on fluorescence intensity changes of paper@QDs@PC-MIPs. (b) Fluorescent quenching changes of paper@QDs@PCMIPs within $30 \mathrm{~min}$. The excited light wavelength and the emission wavelength were 396 and $550 \mathrm{~nm}$, respectively $(n=5)$. 
(a)

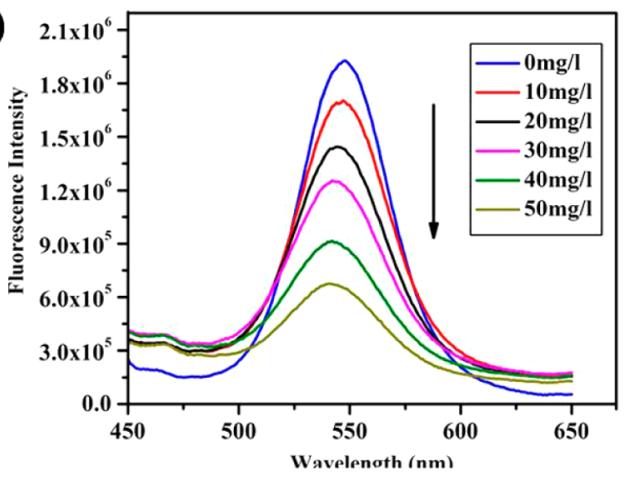

(b)

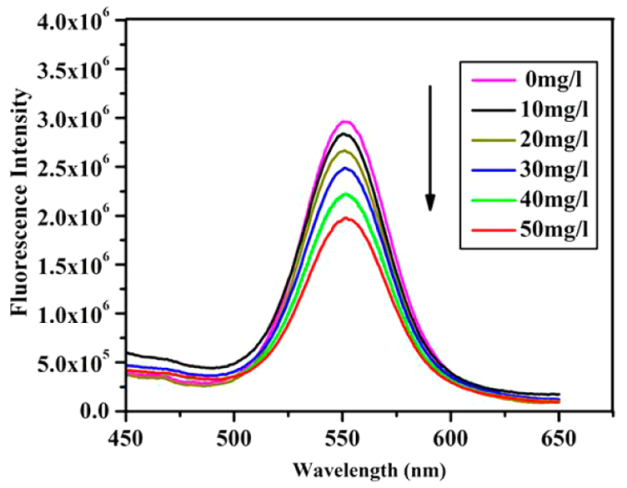

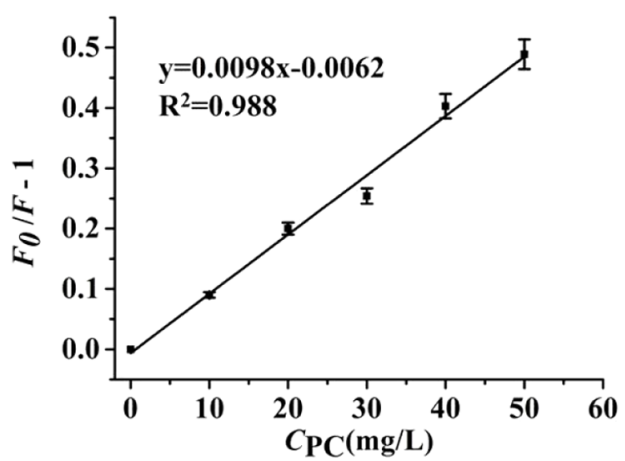

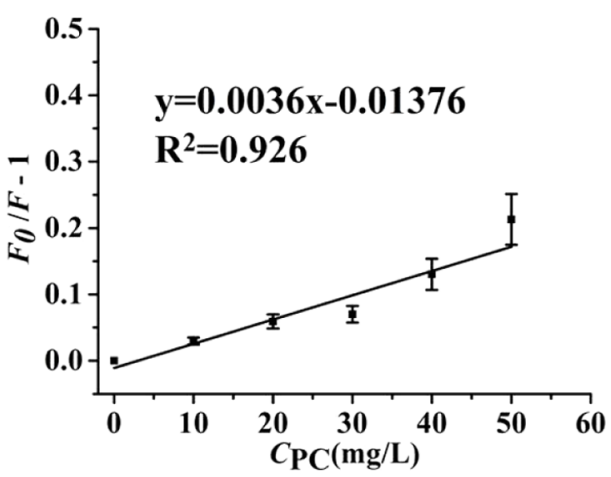

Figure 5. (a) Calibration curve of fluorescence spectra of paper@QDs@PC-MIPs. The concentration of phycocyanin was spiked at 10, 20, 30, 40, and $50 \mathrm{mg} / \mathrm{L}$. (b) Calibration curve of fluorescence spectra of paper@QDs@PC-NIPs. The inset graphs show corresponding Stern-Volmer plots of the molecularly imprinted polymer (MIP) and nonimprinted polymer (NIP). The experimental conditions were $\mathrm{pH}=7.0$ under room temperature. The excited light wavelength and the emission wavelength were 396 and $550 \mathrm{~nm}$, respectively, and the slit widths of emission and excitation were 5 $\mathrm{nm}(n=5)$.

that all the PC was absorbed by the sensors if the concentration of $\mathrm{PC}$ is lower than $150 \mathrm{mg} / \mathrm{L}$ (displayed in Figure S8). If the phycocyanin protein is lower than $50 \mathrm{mg} / \mathrm{L}$, the PC could be quantitatively detected in the linear range on our paper@ QDs@PC-MIP $\mu$ PADs. Therefore, when the quenching constant remains the same, the phycocyanin concentration shows a positive correlation with the fluorescence quenching value. Phycocyanin concentration can be realized by the change in the fluorescence of phycocyanin detection.

The quenching effect can be achieved by Stem-Volmer equation fitting. ${ }^{43,44}$

$$
\left(F_{0} / F\right)-1=K_{\mathrm{SV}} C_{\mathrm{M}}
$$

where $F_{0}$ and $F$ represent the fluorescence intensity in the absence and presence of quencher, respectively; $C_{M}$ is the template concentration; and $K_{\mathrm{SV}}$ is the quenching constant for the quencher. Therefore, the ratio of $K_{\text {sv,MIPs }}$ to $K_{\text {sv,NIPs }}$ is defined as the imprinting factor (IF), and $\left(F_{0} / F\right)-1$ is defined as the quenching amount. IF is one of the important parameters for characterization of molecularly imprinted polymer selectivity.

For qualitative detection of phycocyanin on $3 \mathrm{D}$ origami PQP-MIPs $\mu$ PADs, the fluorescence performance of the phycocyanin was strongly dependent on variables such as $\mathrm{pH}$, response time, and the fluorescence stability. These relevant variables are discussed below. As shown in Figure 4a, when the $\mathrm{pH}$ value was less than 6.0 , the changes in fluorescence intensity were lower. The fluorescence intensity increased greatly with the increase in $\mathrm{pH}$ value from 6 to 7 , and the signal reached a maximum at $\mathrm{pH} 7$. When $\mathrm{pH}$ value was higher than
7.5 , the interaction between the template phycocyanin and the imprinted shells decreased, leading to a dramatic decline in the signal. Considering application in actual samples, $0.01 \mathrm{~mol} / \mathrm{L}$ PBS buffer solution with $\mathrm{pH}=7.0$ was chosen for further experiments.

To evaluate accessibility to binding sites, dynamic adsorption was also investigated over $30 \mathrm{~min}$. As seen from Figure 4b, the fluorescence intensity at $550 \mathrm{~nm}$ decreased within $25 \mathrm{~min}$ when the concentration of $\mathrm{PC}$ was $100 \mathrm{mg} / \mathrm{L}$, after which the adsorption curve plateaued for the remaining $25 \mathrm{~min}$. Therefore, $25 \mathrm{~min}$ was chosen as the response time for further experiments.

The performance of the developed PQP-MIPs $\mu$ PADs for quantitative determination of phycocyanin was further investigated. The response of the PQP-MIPs assay to increasing amounts of phycocyanin is illustrated in Figure 5a. A linear increase in response for phycocyanin amounts ranging 10-50 $\mathrm{mg} / \mathrm{L}$ corresponds to a dynamic range of 1 order of magnitude. As displayed, the fluorescence intensities decreased dramatically with the increase in phycocyanin concentration, and this phenomenon contributed to the quenching effect. The error bars indicated small RSDs for five repeated measurements $\left(R^{2}\right.$ $=0.988$ ). The limit of detection (LOD) of the assay without any amplification steps was found to be $2 \mathrm{mg} / \mathrm{L}$ (calculated from $3 \sigma$ ). The RSD of measurements was $4.56 \%$ calculated from five independent experiments. The paper@QDs@PCMIPs displayed a relatively sensitive response for phycocyanin. As shown in Figure S9, fluorescence values were determined for 20 different positions on the same piece of paper and the RSD of 4.7\% showed satisfactory reproducibility of paper@QDs@ 
PC-MIPs. Negative controls were also performed in a similar manner using nonimprinted polymers instead of the phycocyanin-imprinted polymers. The same amount of $30 \mu \mathrm{L}$ DI water was added dropwise to the detection area to avoid the humidity change. Compared with the NIP results (Figure 5b), in the same concentration range the fluorescence intensities with the MIPs decreased significantly, indicating that specific recognition sites with predetermined selectivity for phycocyanin were formed in the PQP-MIPs $\mu$ PADs. In addition, we implemented fluorescence detection for five different paper@ QDs@PC-MIPs $\mu$ PADs over 7 days. As shown in Figure S10, there were no obvious differences between the results and it indicated storage stability for the paper devices.

Selectivity and Analysis of Real Environmental Water Samples. To evaluate the specific recognition ability of paper@QDs@PC-MIPs for phycocyanin, several proteins$\mathrm{PE}, \mathrm{BSA}, \mathrm{BHB}$, and spirulina-were chosen for the selectivity experiments. As seen from Figure 6, the amount of fluorescence

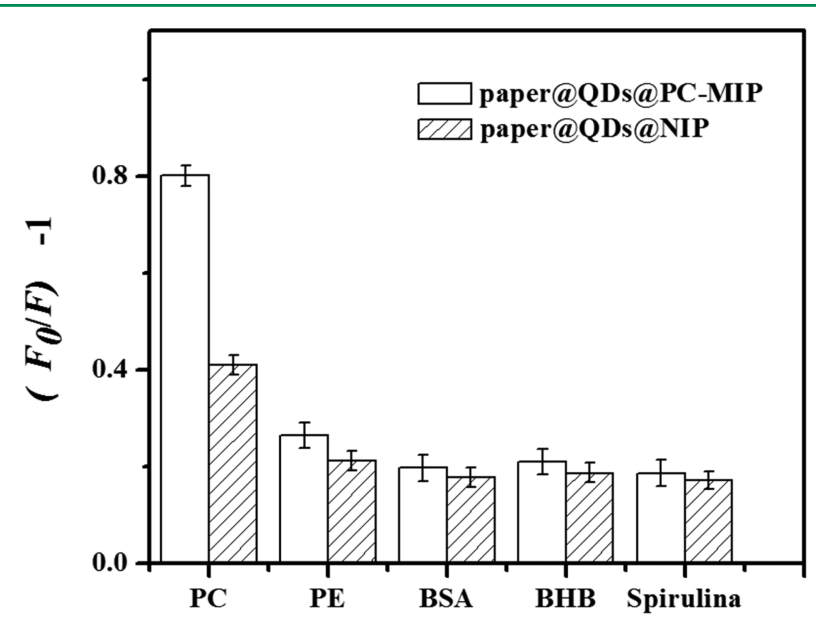

Figure 6. Selectivity of paper@QDs@PC-MIPs and paper@QDs@ NIPs for solutions of different proteins (phycocyanin [PC], phycoerythrin [PE], BSA, BHB, and spirulina) at equal concentrations. The concentration of molecularly imprinted polymers or nonimprinted polymers was $10 \mathrm{mg} / \mathrm{mL}(n=7)$.

quenching of the paper@QDs@PC-MIPs was the largest for phycocyanin, followed by phycoerythrin and spirulina powder. This phenomenon was mainly due to the very similar structures of phycoerythrin and phycocyanin. Moreover, spirulina powder contained a certain amount of phycocyanin and phycoerythrin, and therefore the quenching effect was observed. BSA and BHB have a different space configuration and molecular mass. They may form hydrogen bonds with functional monomer APTES, but are not complementary to the recognition sites that significantly affect the induced fluorescence. Therefore, the PQP-MIPs $\mu$ PADs showed high selectivity toward the template phycocyanin.

Finally, real water samples were used to validate the practical applicability of the PQP-MIPs $\mu$ PADs for detection of phycocyanin. Lake water samples and seawater samples were taken from Yantai University and the fisherman's wharf of the Yellow Sea located in the coastal zone area of Yantai City, respectively. All water samples were filtered through $0.45-\mu \mathrm{m}$ PTFE syringe filters (Phenomenex, USA) with $\mathrm{Na}_{2} \mathrm{HPO}_{4}-$ $\mathrm{NaH}_{2} \mathrm{PO}_{4}$ buffer solution to adjust $\mathrm{pH}$ to 6.5-7.5. The seawater samples were diluted 100 -fold before use. Different concentrations of phycocyanin $(10,20,30 \mathrm{mg} / \mathrm{L})$ were spiked into the water samples without any treatment. The experiment was carried out five times with addition of the standard phycocyanin to the samples. As illustrated in Table 1,

Table 1. Recovery of Spiked Phycocyanin in Lake Water and Seawater Detected Using Paper@QDs@PC-MIPs $\mu$ PADs ${ }^{a}$

$\begin{array}{lcccr}\text { sample } & \begin{array}{c}\text { added } \\ (\mathrm{mg} / \mathrm{L})\end{array} & \begin{array}{c}\text { value }\left(F_{0} /\right. \\ F)\end{array} & \begin{array}{c}\text { detected } \\ (\mathrm{mg} / \mathrm{L})\end{array} & \begin{array}{c}\text { recovery RSD } \\ (\%)\end{array} \\ \text { Seawater } & 10 & 0.091 & 9.91 & 99.1 \pm 5.7 \\ & 20 & 0.201 & 21.14 & 105.7 \pm 4.5 \\ & 30 & 0.271 & 28.29 & 94.3 \pm 3.6 \\ \text { Lake water } & 10 & 0.085 & 9.31 & 93.1 \pm 5.1 \\ & 20 & 0.209 & 21.34 & 106.7 \pm 4.3 \\ & 30 & 0.279 & 29.10 & 97.1 \pm 3.3\end{array}$

${ }^{a}$ Data show detected values and relative standard deviations (RSD, \%, $n=5$ ) for spiked phycocyanin.

satisfactory results were obtained with $94.3-105.7 \%$ recovery and RSD of $3.6-5.7 \%$ for the spiked seawater samples, and $93.1-106.7 \%$ recovery with RSD of $3.3-5.1 \%$ for the spiked lake water samples, respectively. The results demonstrated that our newly developed fluorescent sensor could be successfully used for the detection of phycocyanin in environmental water samples.

\section{CONCLUSIONS}

In this study, we proposed a novel kind of quantum dot wrapped with molecularly imprinted polymers on $3 \mathrm{D}$ origami paper-based microfluidic chip devices that provided a robust and facile route to detect phycocyanin. It can graft quantum dots on the paper through synthesizing paper@QDs@PCMIPs components and realizing the liquid phase of QDs@ MIPs being transferred to the solid-phase paper base. To the best of our knowledge, this is the first demonstration of the concept of combining QDs and MIPs directly on paper's cellulose and assembling it into the origami paper microfluidic devices. The approach offers many attractive advantages such as portability, disposability, low cost, and user-friendly protocol due to the paper's unique characteristics.

The method showed good performance with reproducible results. Successful analysis of real samples of phycocyanin in local lake water and seawater showed good sensitivity and reliability. Moreover, the $3 \mathrm{D}$ origami $\mu \mathrm{PADs}$ device shows great potential and versatility for detection of other different kinds of proteins using appropriate template molecule.

\section{ASSOCIATED CONTENT}

\section{Supporting Information}

The Supporting Information is available free of charge on the ACS Publications website at DOI: 10.1021/acssensors.6b00664.

Experimental details and supporting photographs and figures (PDF)

\section{AUTHOR INFORMATION}

\section{Corresponding Authors}

*E-mail: jbchoo@hanyang.ac.kr.

*E-mail: lxchen@yic.ac.cn.

ORCID

Jaebum Choo: 0000-0003-3864-6459

Lingxin Chen: 0000-0002-3764-3515 


\section{Author Contributions}

${ }^{\#} \mathrm{~B}$. Li and $\mathrm{Z}$. Zhang contributed equally to this work.

\section{Notes}

The authors declare no competing financial interest.

\section{ACKNOWLEDGMENTS}

This work was financially supported by the National Defense Science and Technology Innovation Project of Chinese Academy of Sciences (CXJJ-16M254), the Science and Technology Development Plan of Yantai (2015ZH087), the Natural Science Research Foundation of China (21205131, 41406125), the Fundamental Research Funds for the Central Universities in China (GK201603099), Shanxi Province Agricultural Science and Technology Innovation and Key Project (2016NY-181). This work was also partially supported by the Korea Health Industry Development Institute (KHIDI) funded by the Ministry of Health and Welfare, Korea (HI16C2129).

\section{REFERENCES}

(1) Johnk, K. D.; Huisman, J.; Sharples, J.; Sommeijer, B.; Visser, P. M.; Stroom, J. M. Summer heatwaves promote blooms of harmful cyanobacteria. Global. Change. Biol. 2008, 14, 495-512.

(2) Metcalf, J. S.; Banack, S. A.; Lindsay, J.; Morrison, L. F.; Cox, P. A.; Codd, G. A. A neurotoxic amino acid with other cyanobacterial toxins in British waterbodies. Environ. Microbiol. 2008, 10, 702-708.

(3) Tyler, A. N.; Hunter, P. D.; Carvalho, L.; Codd, G. A.; Elliott, A.; Ferguson, C. A.; Hanley, N. D.; Hopkins, D. W.; Maberly, S. C.; Mearns, K. J.; Scott, E. M. Strategies for monitoring and managing mass populations of toxic cyanobacteria in recreational waters: a multiinterdisciplinary approach. Environ. Health 2009, 8, S11.

(4) Jiao, Y. Y.; Chen, Q. K.; Chen, X.; Wang, X.; Liao, X. W.; Jiang, L. J.; Wu, J.; Yang, L. Y. Sci. Occurrence and transfer of a cyanobacterial neurotoxin beta-methylamino-L-alanine within the aquatic food webs of Gonghu Bay (Lake Taihu, China) to evaluate the potential human health risk. Sci. Total Environ. 2014, 468, 457-463.

(5) Al-Sammak, M. A.; Hoagland, K. D.; Cassada, D.; Snow, D. D. Co-occurrence of the Cyanotoxins BMAA, DABA and Anatoxin-a in Nebraska Reservoirs, Fish, and Aquatic Plants. Toxins 2014, 6, 488508.

(6) Cox, P. A.; Banack, S. A.; Murch, S. J.; Rasmussen, U.; Tien, G.; Bidigare, R. R.; Metcalf, J. S.; Morrison, L. F.; Codd, G. A.; Bergman, B. Proc. Natl. Acad. Sci. U. S. A. 2005, 102, 5074-5078.

(7) Codd, G. A.; Morrison, L. F.; Metcalf, J. S. Cyanobacterial toxins: risk management for health protection. Toxicol. Appl. Pharmacol. 2005, 203, 264-272.

(8) Ledreux, A.; Thomazeau, S.; Catherine, A.; Duval, C.; Yepremian, C.; Marie, A.; Bernard, C. Evidence for saxitoxins production by the cyanobacterium Aphanizomenon gracile in a French recreational water body. Harmful Algae 2010, 10, 88-97.

(9) Nagaoka, S.; Shimizu, K.; Kaneko, H.; Shibayama, F.; Morikawa, K.; Kanamaru, Y.; Otsuka, A.; Hirahashi, T.; Kato, T. A novel protein C-phycocyanin plays a crucial role in the hypocholesterolemic action of Spirulina platensis concentrate in rats. J. Nutr. 2005, 135, 24252430.

(10) Hunter, P. D.; Tyler, A. N.; Carvalho, L.; Codd, G. A.; Maberly, S. C. Hyperspectral remote sensing of cyanobacterial pigments as indicators for cell populations and toxins in eutrophic lakes. Remote. Sens. Environ. 2010, 114, 2705-2718.

(11) Simis, S. G. H.; Peters, S. W. M.; Gons, H. J. Remote sensing of the cyanobacterial pigment phycocyanin in turbid inland water. Limnol. Oceanogr. 2005, 50, 237-245.

(12) Zhang, Z.; Li, J.; Wang, X.; Shen, D.; Chen, L. ACS. Quantum Dots Based Mesoporous Structured Imprinting Microspheres for the Sensitive Fluorescent Detection of Phycocyanin. ACS Appl. Mater. Interfaces 2015, 7, 9118-9127.
(13) Zhang, Z.; Li, J.; Fu, J.; Chen, L. Fluorescent and magnetic dualresponsive coreshell imprinting microspheres strategy for recognition and detection of phycocyanin. RSC Adv. 2014, 4, 20677-20685.

(14) Martinez, A. W.; Phillips, S. T.; Butte, M. J.; Whitesides, G. M. Patterned paper as a platform for inexpensive, low-volume, portable bioassays. Angew. Chem., Int. Ed. 2007, 46, 1318-1320.

(15) Liu, H.; Crooks, R. M. Three-Dimensional Paper Microfluidic Devices Assembled Using the Principles of Origami. J. Am. Chem. Soc. 2011, 133, 17564-17566.

(16) Cuartero, M.; Crespo, G. A.; Bakker, E. Paper-Based Thin-Layer Coulometric Sensor for Halide Determination. Anal. Chem. 2015, 87, 1981-1990.

(17) Li, B.; Fu, L.; Zhang, W.; Feng, W.; Chen, L. Portable paperbased device for quantitative colorimetric assays relying on light reflectance principle. Electrophoresis 2014, 35, 1152-1159.

(18) Ding, J.; Li, B.; Chen, L.; Qin, W. A Three-dimensional Origami Paper Device for Potentiometric Biosensing. Angew. Chem., Int. Ed. 2016, 55, 13033-13037.

(19) Nie, Z. H.; Nijhuis, C. A.; Gong, J. L.; Chen, X.; Kumachev, A.; Martinez, A. W.; Narovlyansky, M.; Whitesides, G. M. Electrochemical sensing in paper-based microfluidic devices. Lab Chip 2010, 10, 477483.

(20) Cuartero, M.; Crespo, G. A.; Bakker, E. Thin layer samples controlled by dynamic electrochemistry. Chimia 2015, 69, 203-206.

(21) Yu, J. H.; Ge, L.; Huang, J. D.; Wang, S. M.; Ge, S. G. Microfluidic paper-based chemiluminescence biosensor for simultaneous determination of glucose and uric acid. Lab Chip 2011, 11, $1286-1291$.

(22) Li, B.; Zhang, W.; Chen, L.; Lin, B. A fast and low-cost spray method for prototyping and depositing surface-enhanced Raman scattering arrays on microfluidic paper based device. Electrophoresis 2013, 34, 2162-2168.

(23) Zhang, W.; Li, B.; Chen, L.; Wang, Y.; Gao, D.; Ma, X.; Wu, A. Brushing, a simple way to fabricate SERS active paper substrates. Anal. Methods 2014, 6, 2066-2071.

(24) Qu, L. L.; Song, Q. X.; Li, Y. T.; Peng, M. P.; Li, D. W.; Chen, L. X.; Fossey, J. S.; Long, Y. T. Fabrication of bimetallic microfluidic surface-enhanced Raman scattering sensors on paper by screen printing. Anal. Chim. Acta 2013, 792, 86-92.

(25) Cheng, C.-M.; Martinez, A. W.; Gong, J.; Mace, C. R.; Phillips, S. T.; Carrilho, E.; Mirica, K. A.; Whitesides, G. M. Paper-Based ELISA. Angew. Chem., Int. Ed. 2010, 49, 4771-4774.

(26) Martinez, A. W.; Phillips, S. T.; Whitesides, G. M.; Carrilho, E. Diagnostics for the Developing World: Microfluidic Paper-Based Analytical Devices. Anal. Chem. 2010, 82, 3-10.

(27) Wang, L.; Xu, C.; Zhu, Y. J.; Yu, Y.; Sun, N.; Zhang, X. Q.; Feng, K.; Qin, J. H. Human induced pluripotent stem cell-derived beating cardiac tissues on paper. Lab Chip 2015, 15, 4283-4290.

(28) Li, X.; Liu, X. Y. A Microfluidic Paper-Based Origami Nanobiosensor for Label-Free, Ultrasensitive Immunoassays. Adv. Healthcare Mater. 2016, 5, 1326-1335.

(29) Wei, X. F.; Tian, T.; Jia, S. S.; Zhu, Z.; Ma, Y. L.; Sun, J. J.; Lin, Z. Y.; Yang, C. J. Microfluidic Distance Readout Sweet Hydrogel Integrated Paper Based Analytical Device (mu DiSH-PAD) for Visual Quantitative Point-of-Care Testing. Anal. Chem. 2016, 88, 2345-2352.

(30) Yan, J. X.; Yan, M.; Ge, L.; Ge, S. G.; Yu, J. H. An origami electrochemiluminescence immunosensor based on gold/graphene for specific, sensitive point-of-care testing of carcinoembryonic antigen. Sens. Actuators, B 2014, 193, 247-254.

(31) Li, W. P.; Li, L.; Li, S.; Wang, X.; Li, M.; Wang, S. W.; Yu, J. H. $3 \mathrm{D}$ origami electrochemiluminescence immunodevice based on porous silver-paper electrode and nanoporous silver double-assisted signal amplification. Sens. Actuators, B 2013, 188, 417-424.

(32) Tian, T.; Wei, X. F.; Jia, S. S.; Zhang, R. H.; Li, J. X.; Zhu, Z.; Zhang, H. M.; Ma, Y. L.; Lin, Z. Y.; Yang, C. J. Integration of target responsive hydrogel with cascaded enzymatic reactions and microfluidic paper-based analytic devices (mu PADs) for point-of-care testing (POCT). Biosens. Bioelectron. 2016, 77, 537-542. 
(33) Choi, S.; Kim, S. K.; Lee, G. J.; Park, H. K. Paper-based 3D microfluidic device for multiple bioassays. Sens. Actuators, B 2015, 219, 245-250.

(34) Liana, D. D.; Raguse, B.; Gooding, J. J.; Chow, E. Recent Advances in Paper-Based Sensors. Sensors 2012, 12, 11505-11526.

(35) Li, X.; Ballerini, D. R.; Shen, W. A perspective on paper-based microfluidics: Current status and future trends. Biomicrofluidics 2012, 6, 011301.

(36) Chen, L. X.; Xu, S. F.; Li, J. H. Recent advances in molecular imprinting technology: current status, challenges and highlighted applications. Chem. Soc. Rev. 2011, 40, 2922-2942.

(37) Chen, L.; Wang, X.; Lu, W.; Wu, X.; Li, J. Molecular imprinting: perspectives and applications. Chem. Soc. Rev. 2016, 45, 2137-2211.

(38) Noor, M. O.; Krull, U. J. Camera-Based Ratiometric Fluorescence Transduction of Nucleic Acid Hybridization with Reagentless Signal Amplification on a Paper-Based Platform Using Immobilized Quantum Dots as Donors. Anal. Chem. 2014, 86, 1033110339.

(39) Zhang, Z.; Li, J. H.; Fu, L. W.; Liu, D. Y.; Chen, L. Magnetic molecularly imprinted microsensor for selective recognition and transport of fluorescent phycocyanin in seawater. J. Mater. Chem. A 2015, 3, 7437-7444.

(40) Wang, S.; Ge, L.; Li, L.; Yan, M.; Ge, S.; Yu, J. Molecularly imprinted polymer grafted paper-based multi-disk micro-disk plate for chemiluminescence detection of pesticide. Biosens. Bioelectron. 2013, $50,262-268$.

(41) Ge, L.; Wang, S.; Yu, J.; Li, N.; Ge, S.; Yan, M. Molecularly Imprinted Polymer Grafted Porous Au-Paper Electrode for an Microfluidic Electro-Analytical Origami Device. Adv. Funct. Mater. 2013, 23, 3115-3123.

(42) Noipa, T.; Tuntulani, T.; Ngeontae, W. $\mathrm{Cu}^{2+}$-modulated cysteamine-capped $\mathrm{CdS}$ quantum dots as a turn-on fluorescence sensor for cyanide recognition. Talanta 2013, 105, 320-326.

(43) Xu, S.; Lu, H.; Li, J.; Song, X.; Wang, A.; Chen, L.; Han, S. Dummy Molecularly Imprinted Polymers-Capped CdTe Quantum Dots for the Fluorescent Sensing of 2,4,6-Trinitrotoluene. ACS Appl. Mater. Interfaces 2013, 5, 8146-8154.

(44) Cheng, P. P. H.; Silvester, D.; Wang, G. L.; Kalyuzhny, G.; Douglas, A.; Murray, R. W. Dynamic and static quenching of fluorescence by 1-4 nm diameter gold monolayer-protected clusters. J. Phys. Chem. B 2006, 110, 4637-4644. 